\title{
Verschlechterung der Lebensqualität von Patienten mit Morbus Hodgkin in den folgenden 5 Jahren nach abgeschlossener Therapie: Langzeitdaten aus den Studien HD13, HD14 und HD15
}

\author{
Dirk Vordermark ${ }^{1}$ \\ Angenommen: 8. Februar 2021 / Online publiziert: 22. März 2021 \\ (c) Der/die Autor(en) 2021
}

\begin{abstract}
Hintergrund Der stadien- und risikoadaptierte Einsatz multimodaler Therapiekonzepte erzielt beim Morbus Hodgkin bekanntermaßen hohe Heilungsraten. Die Langzeitüberlebenden sind jedoch in vielen Bereichen ihrer Lebensqualität beeinträchtigt. Dies beinhaltet spezifische Symptomkomplexe wie Fatigue, aber auch Probleme bei der sozialen und beruflichen Integration der häufig bei Therapie noch sehr jungen und somit im Langzeitverlauf immer noch jungen Patienten. Die Therapieoptimierungsstudien der Deutschen Hodgkin Studiengruppe (DHSG) zielten auf eine Verbesserung des Profils zwischen onkologischer Tumorkontrolle (z. B. gemessen als progressionsfreies Überleben) und Spätfolgen der Therapie ab, z.B. durch Weiterentwicklung der Chemotherapiekonzepte und Modifikation der Strahlentherapie bezüglich Zielvolumen und Dosierung, teilweise auch durch einen Verzicht auf die Radiotherapie. Aktuell wurde nun ein sehr umfangreicher Datensatz zur Lebensqualität im Langzeitverlauf bis fünf Jahre nach Therapieende aus den Studien HD13, HD14 und HD15 publiziert, der eine differenzierte Betrachtung der Entwicklung definierter Bereiche der Lebensqualität wie auch die Identifikation von Risikofaktoren einschließlich des Zusammenhangs mit einzelnen Therapiekonzepten erlaubt.
\end{abstract}

Originalpublikation Kreissl S, Müller H, Goergen H, Meissner J, Topp M, Sökler M, Markova J, Bernhard J, Greil R, von Tresckow B, Behringer K, Rüffer JU, Flechtner HH, Möstl M, Fuchs M, Engert A, Diehl V, Borchmann P (2020) Health-Related Quality of Life in Patients With Hodgkin Lymphoma: A Longitudinal Analysis of the German Hodgkin Study Group. J Clin Oncol 38 (25):2839-2848. https://doi.org/10.1200/JCO.19. 03160. Epub 2020 Jun 23.

\section{Prof. Dr. med. Dirk Vordermark} dvordermark@outlook.de

1 Universitätsklinik für Strahlentherapie, Universitätsklinikum Halle/Saale, Ernst-Grube-Str. 40, 06120 Halle/Saale, Deutschland
Patienten und Methoden Berichtet werden die Daten von 4215 Patienten aus der Studiengeneration HD13 bis HD15 der DHSG mit Beantwortung des Lebensqualitätsfragebogens $\mathrm{zu}$ mindestens einem Zeitpunkt. Eingeschlossen wurden Patienten der Altersgruppe von 18 bis 60 Jahren (bei Therapie), unabhängig vom Remissionsstatus. Die Studie HD13 verglich in der Gruppe der günstigen Frühstadien (Ann Arbor I oder II, ohne Risikofaktoren) vier Arme der Chemotherapie (ABVD, AVD, ABV, AV; die zwei letztgenannten wurden frühzeitig geschlossen). Alle Patienten erhielten eine Involved-field-Radiotherapie mit 30 Gy [1]. Für den Arm ABVD wurde ein progressionsfreies 5-Jahres-Überleben von $93 \%$ erreicht, die anderen Arme waren schlechter. Die Studie HD14 untersuchte in der Gruppe der ungünstigen Frühstadien (I bis II mit definierten Risikofaktoren) die Chemotherapie mit 4 Zyklen ABVD im Vergleich zu 2 Zyklen ABVD+2 Zyklen eskaliertem BEACOPP. Auch hier erhielten alle Patienten eine Involved-field-Radiotherapie mit $30 \mathrm{~Gy}$ [2]. Das progressionsfreie Überleben war mit $95 \%$ signifikant besser nach $2 \times \mathrm{ABVD}+2 \times \mathrm{BEACOPP}$. In HD15 wurden drei Varianten von BEACOPP bei Patienten mit Stadien IIB (mit mediastinalem Bulk) bis IV verglichen. Die Strahlentherapie erfolgte in allen Armen nur auf PET-positive Reste $>2,5 \mathrm{~cm}$ nach Chemotherapie mit $30 \mathrm{~Gy}$, dies betraf lediglich $11 \%$ der Patienten [3]. Der Arm mit 6 Zyklen BEACOPP-eskaliert erreichte das beste progressionsfreie Überleben nach 5 Jahren mit $90 \%$.

Für die aktuell präsentierte Analyse der Lebensqualität wurde der etablierte und validierte Fragebogen European Organization for Research and Treatment of Cancer (EORTC) Quality of Life Questionnaire (QLQ C30) verwendet. Die Befragung erfolgte zu Therapiebeginn, nach zwei bis vier Zyklen Chemotherapie, am Ende der Erstlinienbehandlung (Chemotherapie \pm Radiotherapie) und dann zu den Nachsorgezeitpunkten bis fünf Jahre nach Therapie. Fünf Funktionsbereiche und neun Symptombereiche 
wurden ausgewertet und mit den alters- und geschlechtsadjustierten Werten der deutschen Normalbevölkerung verglichen. Unterschiede von mindestens 10 Punkten (auf der Skala von 0 bis 100) wurden gemäß der üblichen Kriterien als klinisch relevant bewertet.

Ergebnisse Die Rücklaufraten lagen zu Therapiebeginn bei etwa $80 \%$, im zweiten Jahr nach Therapie bei etwa $65 \%$ und im fünften Jahr bei knapp $40 \%$. Starke Selektionseffekte (z. B. Überrepräsentation der Patienten mit weniger Belastung) konnten ausgeschlossen werden. Bei Diagnosestellung wurden in allen Stadiengruppen und Studien (am ausgeprägtesten in höheren Stadien/HD15) starke Belastungen in den Bereichen der emotionalen und sozialen Funktion und bei den Symptomen Fatigue, Schlafstörung und finanzielle Problemen gesehen. Symptome wie Dyspnoe und Appetitverlust waren nur in der mittleren und höheren Stadiengruppe (HD14, HD15) auffällig. Unter der Chemotherapie trat in den meisten Bereichen eine weitere Verschlechterung auf, am dramatischsten in der von den Autoren als „Triade“ bezeichneten Gruppe Fatigue, Rollenfunktion und soziale Funktion.

Im ersten Jahr nach Therapieende und teilweise auch noch bei längerer Nachbeobachtung traten signifikante Erholungen in allen Bereichen auf bis auf die körperliche Funktion. Im betrachteten 5-Jahres-Zeitraum verblieb hier eine deutlich schlechtere Lebensqualität (mit einem klinisch relevanten Delta von 14 bis 22) in allen Funktionsskalen (kognitiv, emotional, sozial), ebenso bei den Symptomen Fatigue, Dyspnoe, Schlafstörung und finanzielle Probleme. Innerhalb der einzelnen Studien konnten keine Unterschiede in der Lebensqualität zwischen den einzelnen Therapiearmen mit unterschiedlichen Chemotherapiekonzepten detektiert werden.

Schlussfolgerungen der Autoren: Bei Langzeitüberlebenden nach Therapie eines Hodgkin-Lymphoms fällt eine umfangreiche und persistierende Verschlechterung der Lebensqualität auf, die weitgehend unabhängig vom gewählten Chemotherapiekonzept ist.

\section{Kommentar}

Der Begriff der Lebensqualität ist in der Onkologie zunehmend aus der Mode gekommen und wird in den letzten Jahren vor allem durch das ,,patient-reported outcome“ (PRO) und dessen elektronische Messung ersetzt. Deren Berücksichtigung im Behandlungsablauf kann die Überlebensprognose der Patienten verbessern [4]. Beiden Konzepten ist gemein, dass die von den Patienten gemachten Angaben (in Papierform oder elektronisch auf einem Smartphone oder Tablet-PC) bei Verwendung validierter Fragebögen ohne ei- ne weitere Zwischenbewertung durch medizinisches Personal in aussagekräftige Scores übersetzt werden.

Die Betrachtung der gesundheitsbezogenen Lebensqualität (,,health-related quality of life“ [HRQOL]) wird dadurch erschwert, dass sie multidimensional ist, also nicht durch einen, sondern durch eine Gruppe von Scores beschrieben wird. Das führt dazu, dass in der (radio-)onkologischen Literatur Lebensqualitätsstudien oft schwer lesbar sind, da unübersichtliche Tabellen generiert werden und durch multiples Testen ,immer irgendetwas signifikant“" ist.

Die Situation der Langzeitüberlebenden nach Therapie des Hodgkin-Lymphoms steht schon lange im Fokus. Denn die Patienten sind überwiegend jung, die Therapien intensiv und die Heilungsraten hoch. Relevante Spätfolgen der Chemotherapie oder der Strahlentherapie wie Zweittumoren oder Kardiotoxizität können nach Jahren bis Jahrzehnten auftreten [5]. Eine Deeskalation der Therapie mit Absenkung der Spätfolgenrisiken bei Erhalt der onkologischen Therapieeffektivität steht deshalb im Fokus der Studienaktivitäten in den jetzt betrachteten erwachsenen Kollektiven. Im pädiatrischen Bereich wird noch stärker sogar die Inkaufnahme einer erhöhten frühen Rezidivrate (bei bestehender Salvage-Option) als akzeptabler Preis für vermiedene Spätfolgen diskutiert [6].

Die vorliegende Arbeit ist zunächst von der Methodik der Auswertung und Ergebnisdarstellung ein Meilenstein. Im Mittelpunkt steht der klinisch relevante Effekt, also das Ausmaß der Abweichung vom Mittelwert der Normalbevölkerung. Dieser wird - trotz der großen Datenmenge - stratifiziert nach Studie, Symptomatik und Zeitpunkt (sowie im Appendix nach Altersgruppe) anschaulich in farbcodierten Heatmaps dargestellt, die für den Strahlentherapeuten intuitiv sind und die „Hot Spots“ der Probleme schnell erkennbar machen.

Die Erkenntnis, dass die Patienten sich von den extremen Belastungen der Therapie zwar partiell erholen, aber in fast keinem Bereich in den ersten fünf Jahren wieder in die Nähe der Normalwerte kommen, ist nicht überraschend. Diese Patienten bedürfen einer Langzeitnachsorge und einige neue Strukturen der letzten Jahre, z. B. die Children-adolescent-young-adult(CAYA)-Einheiten an den Kliniken oder Unterstützungsangebote für junge Erwachsene der Landeskrebsgesellschaften, unterstützen diese Patientengruppe.

Da die Therapieintensität in allen Stadiengruppen auf einen ähnlichen Zielwert titriert wurde (progressionsfreies Überleben 90-95\%), erhalten die Patienten, die aufgrund der Tumorausdehnung schon bei Diagnose die stärkste Einschränkung der Lebensqualität hatten, auch die intensivste Therapie mit den höchsten weiteren Belastungen. Dass innerhalb der einzelnen Studiengenerationen der jeweils beste Studienarm nicht zu einem anderen Lebensqualitätsprofil führte als der jeweilige Vergleichsarm, ist erfreulich, denn 
dieser Befund bestätigt, dass ein früher Vorteil in onkologischen Outcomes nicht mit späteren Belastungen der Lebensqualität erkauft wurde.

Leider ermöglicht die aktuelle Studie keine Betrachtung der oft kontrovers diskutierten Frage, welchen Beitrag in der Behandlung des Hodgkin-Lymphoms die Chemotherapie bzw. die Strahlentherapie zu den Spätfolgen und den Veränderungen der Lebensqualität leistet, denn in keiner der inkludierten Studien wurde bezüglich des Einsatzes der Strahlentherapie randomisiert. Hier werden erst Lebensqualitätsdaten der darauf folgenden Studiengeneration interessante Einblicke liefern. Bisherige Studien zur Lebensqualität von Patienten mit Hodgkin-Lymphomen lassen jedenfalls keinen deutlichen Effekt der einzelnen Therapiemodalitäten ausmachen (Übersicht in [7]).

Wir wissen jedoch aus Langzeitdaten, dass auch die alleinige Strahlentherapie des Morbus Hodgkin (wenngleich mit höheren Dosen, größeren Zielvolumina und heute obsoleten Techniken) klinisch relevante Auswirkungen auf die Lebensqualität haben kann [8]. Inwiefern die heute im adulten oder pädiatrischen Bereich eingesetzten Strahlentherapieverfahren (z. B. 20 Gy mit Involved-node- oder Involvedsite-Konzept) einen detektierbaren Einfluss auf die Lebensqualität haben, wird zukünftig von großem Interesse sein.

\section{Fazit}

Erwachsene Patienten mit Hodgkin-Lymphom, die mit einer Kombination aus Chemotherapie und Involved-fieldStrahlentherapie behandelt wurden, weisen bis zu fünf Jahre nach Therapieabschluss erhebliche Einschränkungen in den meisten Bereichen der Lebensqualität auf. Die Daten verdeutlichen den Unterstützungsbedarf dieser Gruppe von Langzeitüberlebenden. Welchen Einfluss die in den letzten zwei Jahrzehnten etablierten Modifikationen der Strahlentherapieverfahren beim Morbus Hodgkin auf Spätfolgen und Lebensqualität haben, ist aufgrund der unterschiedlichen Studiendesigns aus diesen Daten noch nicht zu entnehmen.

\section{Dirk Vordermark, Halle/Saale}

Funding Open Access funding enabled and organized by Projekt DEAL.

Interessenkonflikt D. Vordermark gibt an, dass kein Interessenkonflikt besteht.

Open Access Dieser Artikel wird unter der Creative Commons Namensnennung 4.0 International Lizenz veröffentlicht, welche die Nutzung, Vervielfältigung, Bearbeitung, Verbreitung und Wiedergabe in jeglichem Medium und Format erlaubt, sofern Sie den/die ursprünglichen Autor(en) und die Quelle ordnungsgemäß nennen, einen Link zur Creative Commons Lizenz beifügen und angeben, ob Änderungen vorgenommen wurden.
Die in diesem Artikel enthaltenen Bilder und sonstiges Drittmaterial unterliegen ebenfalls der genannten Creative Commons Lizenz, sofern sich aus der Abbildungslegende nichts anderes ergibt. Sofern das betreffende Material nicht unter der genannten Creative Commons Lizenz steht und die betreffende Handlung nicht nach gesetzlichen Vorschriften erlaubt ist, ist für die oben aufgeführten Weiterverwendungen des Materials die Einwilligung des jeweiligen Rechteinhabers einzuholen.

Weitere Details zur Lizenz entnehmen Sie bitte der Lizenzinformation auf http://creativecommons.org/licenses/by/4.0/deed.de.

\section{Literatur}

1. Behringer K, Goergen H, Hitz F, Zijlstra JM, Greil R, Markova J, Sasse S, Fuchs M, Topp MS, Soekler M, Mathas S, Meissner J, Wilhelm M, Koch P, Lindemann HW, Schalk E, Semrau R, Kriz J, Vieler T, Bentz M, Lange E, Mahlberg R, Hassler A, Vogelhuber M, Hahn D, Mezger J, Krause SW, Skoetz N, Böll B, von Tresckow B, Diehl V, Hallek M, Borchmann P, Stein H, Eich H, Engert A, German Hodgkin Study Group Swiss Group for Clinical Cancer Research (2015) Omission of dacarbazine or bleomycin, or both, from the ABVD regimen in treatment of early-stage favourable Hodgkin's lymphoma (GHSG HD13): an open-label, randomised, noninferiority trial. Lancet 385(9976): 1418-1427

2. von Tresckow B, Plütschow A, Fuchs M, Klimm B, Markova J, Lohri A, Kral Z, Greil R, Topp MS, Meissner J, Zijlstra JM, Soekler M, Stein H, Eich HT, Mueller RP, Diehl V, Borchmann P, Engert A (2012) Dose-intensification in early unfavorable Hodgkin's lymphoma: final analysis of the German Hodgkin Study Group HD14 trial. J Clin Oncol 30(9):907-913

3. Engert A, Haverkamp H, Kobe C, Markova J, Renner C, Ho A, Zijlstra J, Král Z, Fuchs M, Hallek M, Kanz L, Döhner H, Dörken B, Engel N, Topp M, Klutmann S, Amthauer H, Bockisch A, Kluge R, Kratochwil C, Schober O, Greil R, Andreesen R, Kneba M, Pfreundschuh M, Stein H, Eich HT, Müller RP, Dietlein M, Borchmann P, Diehl V, German Hodgkin Study Group Swiss Group for Clinical Cancer Research (2012) Reduced-intensity chemotherapy and PET-guided radiotherapy in patients with advanced stage Hodgkin's lymphoma (HD15 trial): a randomised, open-label, phase 3 non-inferiority trial. Lancet 379(9828):1791-1799

4. Basch E, Deal AM, Dueck AC, Scher HI, Kris MG, Hudis C, Schrag D (2017) Overall survival results of a trial assessing patient-reported outcomes for symptom monitoring during routine cancer treatment. JAMA 318(2):197-198

5. Machann W, Beer M, Breunig M, Störk S, Angermann C, Seufert I, Schwab F, Kölbl O, Flentje M, Vordermark D (2011) Cardiac magnetic resonance imaging findings in 20-year survivors of mediastinal radiotherapy for Hodgkin's disease. Int J Radiat Oncol Biol Phys 79(4):1117-1123

6. Mauz-Körholz C, Hasenclever D, Dörffel W, Ruschke K, Pelz T, Voigt A, Stiefel M, Winkler M, Vilser C, Dieckmann K, Karlén J, Bergsträsser E, Fosså A, Mann G, Hummel M, Klapper W, Stein H, Vordermark D, Kluge R, Körholz D (2010) Procarbazine-free OEPA-COPDAC chemotherapy in boys and standard OPPA-COPP in girls have comparable effectiveness in pediatric Hodgkin's lymphoma: the GPOH-HD-2002 study. J Clin Oncol 28(23):3680-3686

7. Linendoll N, Saunders T, Burns R, Nyce JD, Wendell KB, Evens AM, Parsons SK (2016) Health-related quality of life in Hodgkin lymphoma: a systematic review. Health Qual Life Outcomes 14(1):114

8. Norum J, Wist EA (1996) Quality of life in survivors of Hodgkin's disease. Qual Life Res 5(3):367-374 